\title{
ED cognition: any decision by anyone at any time
}

\author{
Pat Croskerry, MD, PhD
}

The ability of an emergency department (ED) to meet the needs of its patients depends largely on the resources available. Relevant resources include nurses, physicians, paramedics, specialty backup, care spaces, inpatient bed base, space, equipment, and supplies. All are important to ED function; however, one resource that is seldom considered is cognition, the cognitive capacity of ED staff. Although invisible, it is arguably our most critical resource. Everything that is done for patients-their diagnosis, management, treatment, and disposition-requires decision making that depends on both individual and collective cognition. In the past, we have taken cognition for granted, assuming that if we bring physicians, nurses, paramedics, and patients together, the necessary decision making will occur. However, things may not be quite so simple. In recent years, we have realized that ED cognition is finite, that overtaxing it may compromise patient care, and that we must protect it and use it judiciously. The purpose of this article is to explore how cognition is used (and abused) and the impact this may have on patient safety.

\section{A LABORATORY FOR ERROR}

The ED is a unique environment. It is difficult to imagine any other workplace in which there exists such range of problems, acuity, time pressure and decision density. There are few environments outside medicine that rival its complexity, unpredictability, and potential for causing harm. This high level of complexity is a threat to patient safety, and it is not surprising that the ED has been described as a natural laboratory for human error. ${ }^{1}$

A modified delphian study identified 25 ED processes that serve as potential sources of error and threaten patient safety. 2,3 These fell into two broad groups: processes that are largely under the immediate control of the individual and processes that depend more on system design and function (Figure 1). Patient safety can be compromised by errors in any of these processes, but the greatest impact is likely when individual cognition is compromised. The systemindividual approach described above is a convenient way of looking at ED function, but it should be noted that the two process types are not independent. Considerable overlap exists; at the end of the day, all aspects of the system are designed by individuals. To further complicate matters, consider that each process can be divided into subprocesses. For example, laboratory error may involve five main subprocesses, each of which can be further subdivided. ${ }^{4}$

Another way of looking at the burden of ED complexity on cognition is by classifying its characteristics into error-producing conditions (EPCs), which may be intrinsic or systemic ${ }^{5}$ (Table 1). Intrinsic EPCs are inherent in the practice of emergency medicine and come with the territory; they present the strongest drain on ED cognition. Systemic EPCs are largely due to the way in which the ED is designed and operated. They place considerable burden on ED cognition but may be alleviated to a large extent by good design and management.

Complex interrelated ED processes, combined with intrinsic and systemic EPCs, create an environment that is uniquely challenging and distinctly different from any other in medicine. The remainder of this article focuses on three features that pose particular threats to ED cognition and patient safety: decision making, ED overcrowding, and fatigue and shiftwork.

From the Critical Thinking Program and Division of Medical Education, Dalhousie University, Halifax, NS.

Correspondence to: Dr. Pat Croskerry, Critical Thinking Program, Division of Medical Education, Dalhousie University, Clinical Research Centre, 5849 University Avenue, PO Box 15000, Halifax, NS B3H 4R2; croskerry@eastlink.ca.

This article has been peer reviewed. 


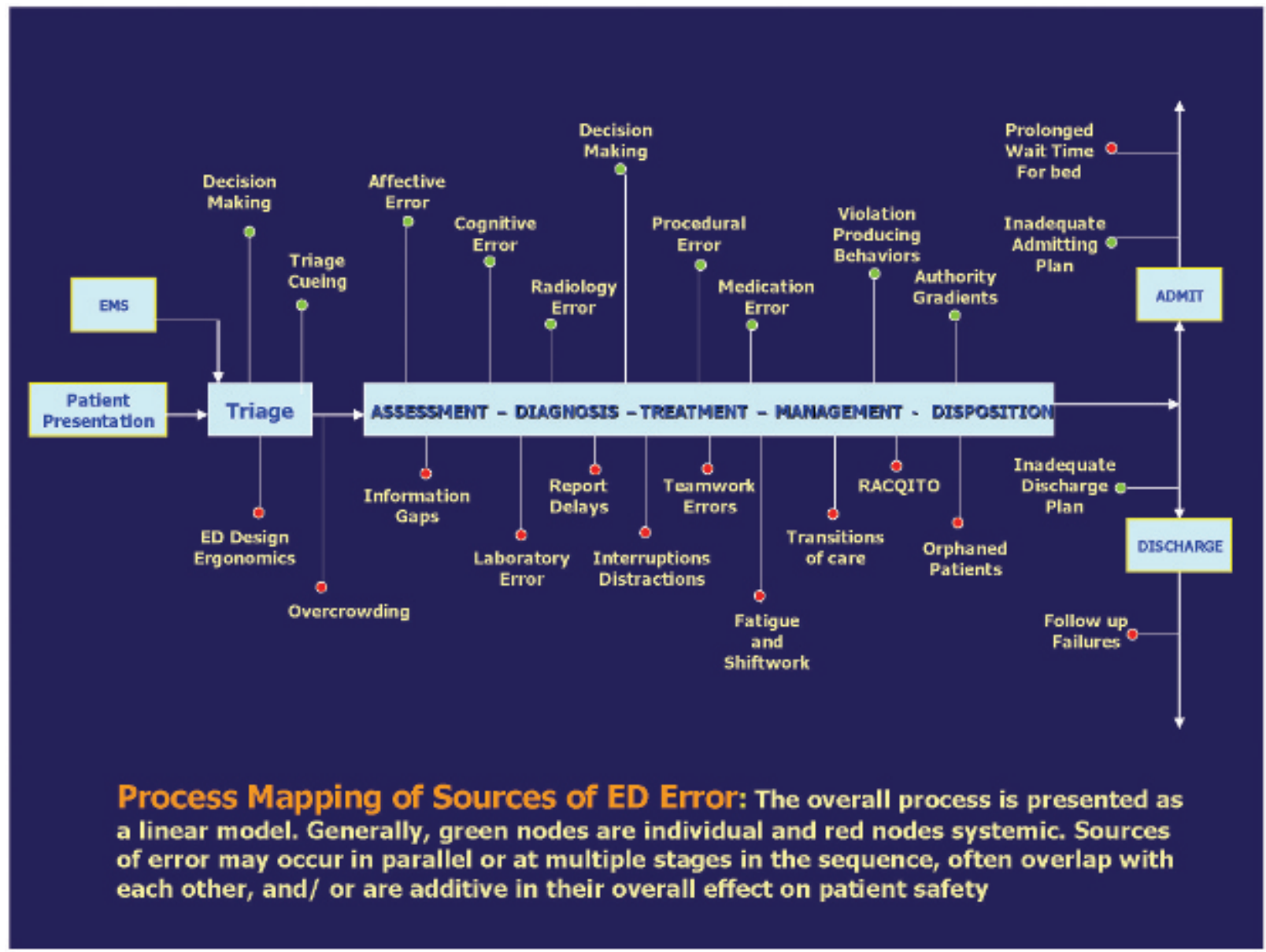

Figure 1. Process mapping of sources of emergency department (ED) error. EMS = emergency medical services; RACQITO = resource availability continuous quality improvement trade off.

\section{DECISION MAKING}

Clinical and nonclinical decision making are the most important processes that happen in an ED. Decisions at the executive management level determine ED staffing levels, coverage hours, bed availability, and other issues that affect ED operations. Decision making by physicians, clerical staff, nurses, paramedics, radiology staff, laboratory staff, and others is part of a distributed cognition that determines ED effectiveness.

In recent years, consensus models have emerged describing the processes of human decision making. The prevailing view is that human cognition occurs via one of two broad mechanisms: the intuitive mode, referred to as System 1 or Type 1 processing, and the analytic mode, referred to as System 2 or Type 2 processing. The literature on these two systems is now protean, and there is little in the way of a working alternative for how human reasoning operates. ${ }^{6-8}$ It is important to understand the model because it applies to all human reasoning and provides explanations for how reasoning and decision making fail and where cognition breaks down. Every decision made by anyone at any time can be described by this model, including all important diagnostic and therapeutic decisions made by emergency physicians. ${ }^{9}$

Diagnostic error in the ED is largely due to defects in procedural knowledge (knowing how to reason) rather than in declarative knowledge (knowing medical facts). Thus, as several studies show, common diagnoses account for more diagnostic failure than rare, esoteric ones do, ${ }^{10-12}$ and the predominant cause of misdiagnosis is cognitive failure, not knowledge deficits. ${ }^{12,13}$ It is now widely accepted that most cognitive failures occur in the intuitive mode of reasoning and are attributed to biased decision making and the use of heuristics. ${ }^{7}$ Although we spend most of our cognitive time in the intuitive mode and make many useful decisions there, it is the cognitive place where most errors occur-some of them catastrophic. Vivid examples in emergency medicine have 


\begin{tabular}{l} 
Table 1. Error-producing conditions in the ED \\
\hline Intrinsic \\
\hline High decision density \\
High levels of diagnostic uncertainty \\
High cognitive load \\
Narrow time windows for assessment \\
Multiple transitions of care \\
Multitasking \\
Interruptions/distractions \\
Low signal to noise ratio \\
Surge phenomena \\
Fatigue \\
Circadian dyssynchronicity \\
Sleep deprivation/debt \\
Novel or infrequently occurring conditions \\
Extrinsic \\
\hline Suboptimal ED design \\
Suboptimal ergonomics \\
High communication load \\
Overcrowding \\
Cognitive overloading \\
Holding admitted patients \\
Production pressures \\
High noise levels \\
Inadequate staffing \\
Poor feedback \\
Limited resources \\
ED = emergency department. \\
\hline
\end{tabular}

been described, ${ }^{14}$ and there are few emergency physicians of any experience who would not be aware of similar cases.

Several important issues emerge from this. The first is that ED physicians need to realize that most of their thinking failures occur in the intuitive mode; therefore, a strong imperative exists to find ways to improve critical thinking and especially intuitive performance.

\section{IMPROVING INTUITIVE PERFORMANCE}

It is a rule of thumb among psychologists that we spend over $90 \%$ of our conscious time in the intuitive mode..$^{15}$ Given that most cognitive error occurs in this mode, it follows that we need to be constantly vigilant about intuitive thinking and decision making. Several strategies have been proposed to mitigate error. ${ }^{16}$ These apply to both learners in the ED and experienced clinicians who strive to become better calibrated in their decision making.

\section{Optimize the environment}

Both Type 1 and Type 2 decisions will be better in a good environment. Favourable environments are supportive and ergonomically sound and provide expert high-level tutoring and mentoring in a collegial setting. Learners should be well rested and well motivated, as should their mentors. Cognitive load, interruptions, and distractions should all be minimized. Many opportunities should be provided for specific practice in the environment in which the learner is seeking to develop expertise; we should proactively seek environments that favour the acquisition of valid intuitions in learners. Remember that unconscious "tacit" learning is ongoing in any environment.

\section{Provide effective feedback}

Good feedback is essential to the acquisition of any skill. It should be as immediate as possible, relevant to the task at hand, accurate, and unambiguous. Simple "good" or "bad" assessments are of less value than those that focus on multiple attributes of performance.

\section{Impose cognitive circuit breakers}

Do not act without thinking. An almost feral vigilance is required to detect personal bias, as well as the biases of team members, that may arise from intuitive processes. Encourage a reflective approach and, specifically, a thoughtful slowing down of interventions where this is feasible. ${ }^{17}$ Impose forcing functions for predictable errors. Encourage skepticism and the habit of routinely challenging without being confrontational. Seek disconfirming evidence rather than confirming what you intuitively believe.

\section{Acknowledge emotions}

Encourage awareness of and insight into emotions. Recognize the cues of visceral arousal and treat emotions as data. Avoid decisions driven by immediate (hot) emotions and consider slowing down or taking a timeout if viscerally aroused. Recognize that positive mood states are associated with relaxation and openness to new ideas but can also lead to error. Nurture your ability to infer emotional state in others (emotional intelligence) and act on this information. 


\section{Explore connections}

Encourage conscious use of narrative and analogy to make connections between different aspects of the problem. Avoid rigid, preprogrammed responses to clinical problems and try to think of what you cannot see rather than what is immediately apparent. Look for relationships and covariation between variables that may not look obviously related. Allow alternatives to stereotypical, logical analysis. Use imaginative strategies to explore connections outside the boundaries in which information is presented and configure problems in different ways. For example, the way in which various solutions to a problem are perceived is often limited by the way the problem is "framed" to us and dissuades us from making connections between, say, abdominal pain and migraine, neurologic symptoms and hypoglycemia, flank pain and a dissecting aneurysm, or cough and pulmonary embolus. Lateral thinking and exploring connections can be productive.

\section{Accept conflict in choice}

Acknowledge the limited rationality of decision making (we rarely know all there is to know about a particular problem) and be willing to leave conditions undiagnosed if true uncertainty exists. Chest pain not yet diagnosed, for example, is preferable to making a specific diagnosis for which there may not yet be sufficient evidence and which later may lead others astray.

\section{Make scientific method intuitive}

Teach about the predictable pitfalls specific to any area and introduce forcing functions to highlight and avoid them. Always consider worst-case scenarios. Embed forcing functions so well that they are engaged reflexively.

\section{COGNITIVE DEBIASING}

Despite the obvious need to strengthen the intuitive mode of human reasoning, a general pessimism has prevailed regarding the likelihood that decision makers can overcome their hardwired and acquired biases. Kahneman has expressed reservations, noting: "The way to block errors that originate in System 1 is simple in principle: recognize the signs that you are in a cognitive minefield, slow down, and ask for reinforcement from System 2." " That reinforcement is provided by the executive override of system 2 (Figure 2), and this is a critical strategy to optimize clinical reasoning. The mark of a well-calibrated thinker is the ability to rise above his or her impulsive "first impression" and apply a more analytic approach when necessary. Several recent books in the popular press have described more proactive approaches to mobilizing the override function. ${ }^{18-20}$

Important features of cognitive debiasing are now taking shape. It must be recognized at the outset that decision making is a flawed process, and we must change our approach to improve. Understanding the dual process theory and its general operating characteristics is a critical first step (Table 2). We should not be seduced by our intuitions but recognize instead that although they may serve us well most of the time, they are where we are at our most vulnerable. Furthermore, we should understand that biases are not a homogeneous group of flawed responses but instead originate from a variety of sources, ${ }^{21}$ each of which may need different approaches to mitigate them. We will need both generic and specific strategies

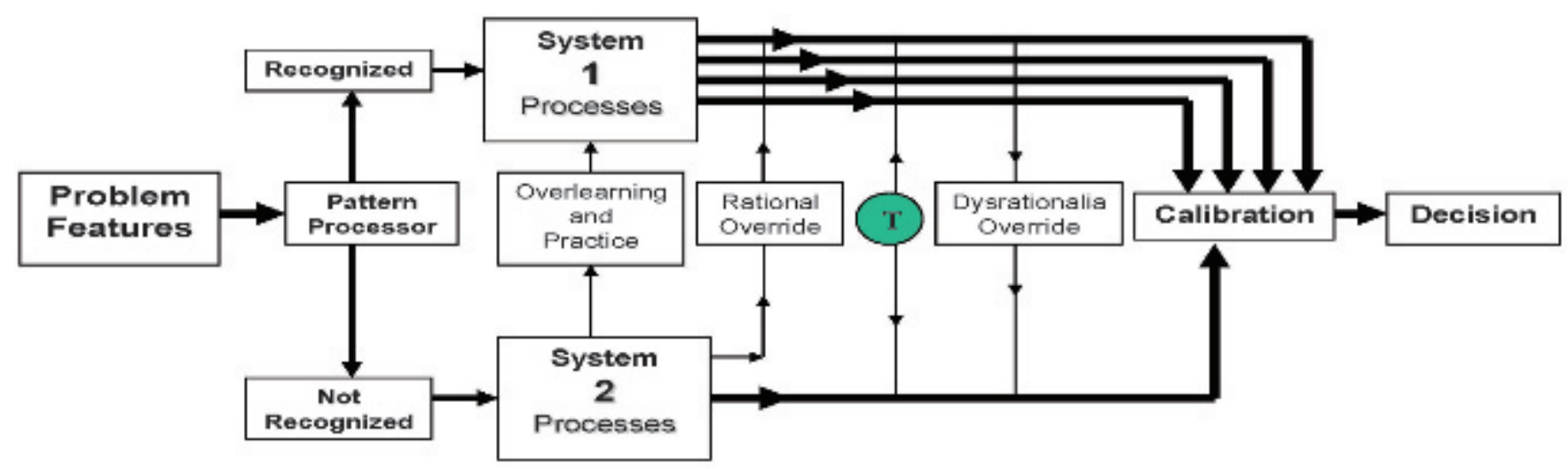

Figure 2. Dual process model for decision making. 
and should expect that repeat interventions will be necessary to maintain change. Neither should we forget those conditions that increase vulnerability to bias: fatigue, sleep deprivation, sleep debt, and overcrowding.

\section{IMPACT OF ED OVERCROWDING}

Overcrowding is a significant source of stress for emergency physicians and leads to unsafe conditions at several levels. Inevitably, it pushes some ED physicians into increased risk-taking behaviour. Long waiting times pressure physicians to make faster decisions and take shortcuts; hence, some patients who might have warranted further observation or even admission may be prematurely discharged. These and other consequences of prolonged ED lengths of stay (related to overcrowding) are associated with increased 7-day morbidity and mortality in patients discharged from the ED. ${ }^{22}$ Overcapacity conditions stretch all resources, leading to a lack of beds and personnel and potentially unsafe care provided in corridors. They generate more ED handovers, increased numbers of care providers involved with each patient (with each successive provider knowing less and less about the patient), and an overall negative impact on nurse and physician well-being.

Most important, from the perspective of this article, is that overcrowding negatively impacts cognition. The tacit assumption is that everyone is busier; therefore, people work harder and make more decisions, but there is more to it than that. Overcrowding not only increases decision density; it also leads to cognitive overload, which changes the nature of decision making (Figure 3). Psychological studies show that cognitive

Table 2. Main features of the dual process model of decision making

System 1 processing is intuitive and fast and works most of the time

We spend most of our time in System 1

Most errors occur in System 1

System 2 processing is analytic, slow, deliberate, and generally more reliable

Repetitive processing in System 2 can result in relegation to System 1

System 2 can override System 1 through the executive override function (cognitive debiasing)

System 1 can override System 2 through dysrationalia

The brain has an automatic tendency to default to System 1

(cognitive miser function)

Toggling can occur between the two systems

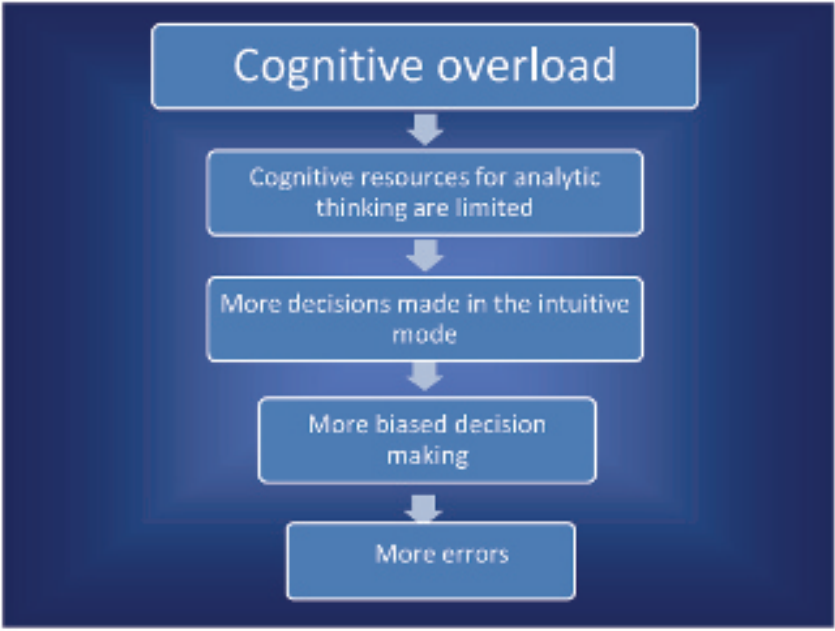

Figure 3. Effects of cognitive overload.

loading selectively interferes with System 2 reasoning, resulting in increased time to make analytic decisions. ${ }^{23}$ Decision makers have an increased tendency to resort to intuitive decision making, where most errors occur. Cognitive loading also increases our tendency to engage the cognitive miser function-to invest less effort in decision making and show less flexibility in toggling between the two modes. ${ }^{24}$ Importantly, a reduced level of executive control means that any effort toward debiasing will be diminished and therefore increase the likelihood of biased reasoning. Thus, cognitive overload compromises ED cognition and adversely affects the quality and safety of decision making.

An overcrowded work environment erodes morale and produces negative affective states in some individuals. These mood states may lead to the "adoption of a low effort information-processing style" 25 demonstrated to result in poorer quality of decision making in health care professionals. ${ }^{26}$ Hogarth described overcrowded EDs as "wicked" environments characterized by poor learning, unreliable or nonexistent feedback, and generally poor milieus for acquiring valid intuitions. ${ }^{16}$ Not only are they adverse environments for physicians, nurses, and paramedics who work there, they are also especially poor for trainees. Hogarth proposed several strategies to improve our intuitive performance (see Improving Intuitive Performance, above).

\section{FATIGUE AND SHIFTWORK}

Shiftwork is an intrinsic EPC of the ED. Given that human injury and illness occur around the clock, most 
EDs are in operation continuously; therefore, disruption of individual circadian rhythms is inevitable. The variety of ways in which these circadian disruptions adversely impact the health of the shiftworker have been reviewed in detail by other researchers. ${ }^{27}$ Most telling, perhaps, is that the World Health Organization has recently added shiftwork to its list of carcinogenic agents. ${ }^{28}$

Importantly, shiftwork adversely impacts cognition and decision making. Table 3 summarizes the main consequences of sleep deprivation, whereas Figure 4 illustrates the complex interplay between sleep deprivation, sleep debt, and fatigue in the shiftworker. Fatigue is distinct from sleep deprivation and sleep debt, but the three are often considered together, and they clearly interact, contributing to cognitive failure. Even without sleep deprivation or sleep debt, simply being awake beyond about 16 hours leads to cognitive decline. ${ }^{29}$ Also, within the normal wake period, there are now clear demonstrations in nonmedical settings that extraneous factors significantly impact decision making over relatively short periods, ${ }^{30}$ perhaps though decision fatigue.

Sleep deprivation probably constitutes the biggest threat to patient safety. The average cognitive decrement toward the end of a typical nightshift is estimated at about $25 \% . .^{31,32} \mathrm{~A}$ major impact is the loss of cognitive executive function, located mostly in the

\section{Table 3. Summary of effects of sleep deprivation on} cognitive performance

Decline in working (short-term) memory

Impaired performance on attention-intensive tasks

Lapses in concentration

Increased distractibility

Lost information

Increased incidence of microsleeps

Increased errors of omission and commission

Longer reaction time

Decreased psychomotor performance

Impaired learning of cognitive tasks

Deterioration of divergent thinking

Prefrontal cortex-mediated tasks more vulnerable to response suppression errors

Increased likelihood of response perseveration on ineffective solutions

More compensatory effort needed to maintain behavioural effectiveness

Performance decrements as task duration increases

Loss of situational awareness resulting in reduced attention to activities judged to be nonessential

Increased tolerance of risk

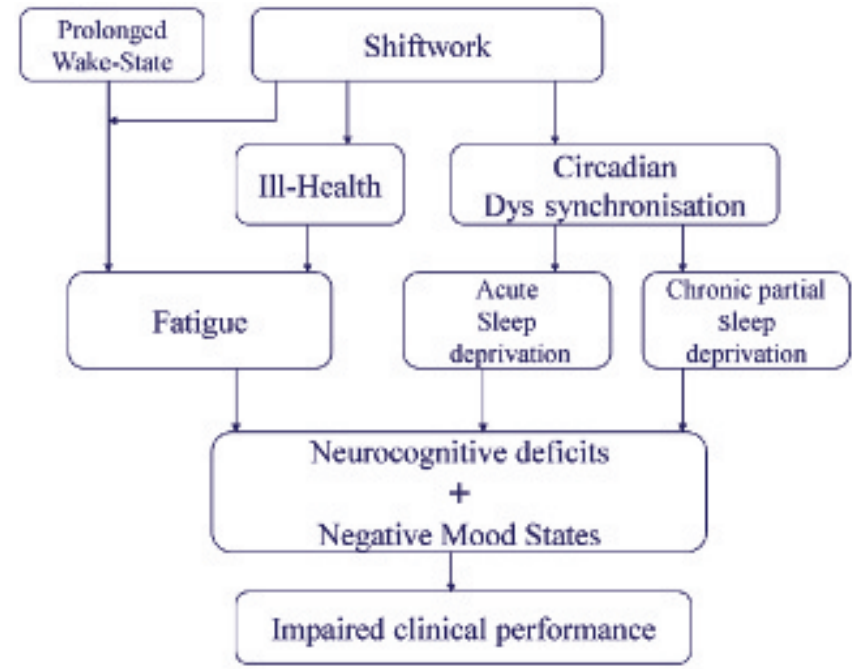

Figure 4. Interrelationships between fatigue, sleep deprivation, and sleep debt.

prefrontal cortex..$^{33}$ As with cognitive overload, this leads to diminished System 2 performance, degraded analytic reasoning, and impaired monitoring and debiasing of System 1. ${ }^{27}$ There is now extensive literature on the adverse impact of sleep deprivation on patient safety, ${ }^{27}$ but there is no easy way around this problem. Shiftwork is a necessary evil, and compromised cognition is inevitable. One key strategy that appears to minimize the impact of shiftwork is casino scheduling, ${ }^{27}$ but it is not always easy to implement at the outset unless there is extensive buy-in from the physician or nursing group. Self-scheduling offers opportunities to ameliorate some of the impact of circadian disruption by accommodating individual differences associated with chronotype, gender, and age. ${ }^{27}$

\section{CONCLUSIONS}

An understanding of ED cognition is important for emergency medicine and patient safety. A central concept is the paradigm of dual process cognition, now a watershed in our understanding of medical decision making. The basic operating characteristics of the dual process approach provide a platform for understanding how critical aspects of ED operation can be compromised and improved. Overcrowding, shiftwork, and fatigue are key examples, but other features, such as interruptions, distractions, attentional capture, ambient conditions, ergonomic shortcomings, and individual differences, are also better understood in the context of the dual processing model. As care 
providers, we must remind ourselves that we operate in a cognitive minefield and that a better understanding of ED cognition is our most precious resource for navigating it.

Competing interests: None declared.

Keywords: decision making, medical error, safety

\section{REFERENCES}

1. Bogner MS, editor. Human error in medicine. Hillsdale (NJ): Lawrence Erlbaum Associates; 1994.

2. Croskerry P, Shapiro M, Perry S, Vinen J. Process improvement and error in the emergency department. Emerg Med Australas 2004;16 Suppl 1:A44.

3. Croskerry P, Shapiro M, Perry S, Wears R. Process improvement and error management in the ED. In: Marx JA, Hockberger R, Walls R, (editors). Rosen's emergency medicine: concepts and clinical practice. 6th ed. St. Louis: Mosby Inc; 2006. p. 3119-26.

4. Croskerry P. Laboratory error and emergency medicine. In: Croskerry P, Cosby KS, Schenkel S, Wears R, (editors). Patient safety in emergency medicine. Philadelphia: Lippincott Williams \& Wilkins; 2008. p. 119-25.

5. Croskerry P, Wears RL. Safety errors in emergency medicine. In: Markovchick VJ, Pons PT, (editors). Emergency medicine secrets. 3rd ed. Philadelphia: Hanley and Belfus; 2003. p. 29-37.

6. Croskerry P. Clinical cognition and diagnostic error: applications of a dual process model of reasoning. $A d v$ Health Sci Educ 2009;14:27-35, doi:10.1007/s10459-0099182-2.

7. Croskerry P. Perspectives on diagnostic error and patient safety. Healthc Papers 2012;15:50-6.

8. Kahneman D. Thinking fast and slow. Doubleday; 2011.

9. Croskerry P. A universal model for diagnostic reasoning. Acad Med 2009;84:1022-8, doi:10.1097/ACM.0b013e3181ace703.

10. Singh H, Thomas EJ, Khan MM, Petersen LA. Identifying diagnostic errors in primary care using an electronic screening algorithm. Arch Intern Med 2007;167:302-8, doi:10.1001/archinte.167.3.302.

11. Schiff G, Hasan O, Kim S, et al. Diagnostic error in medicine. Arch Intern Med 2009;169:1881-7, doi:10.1001/ archinternmed.2009.333.

12. Zwaan L, de Bruijne M, Wagner C, et al. Patient record review of the incidence, consequences, and causes of diagnostic adverse events. Arch Intern Med 2010;170:101521, doi:10.1001/archinternmed.2010.146.

13. Graber ML, Franklin N, Gordon R. Diagnostic error in internal medicine Arch Intern Med 2005;165:1493-9, doi: $\underline{10.1001 / \text { archinte.165.13.1493. }}$.

14. Campbell SA, Croskerry $\mathrm{P}$, Bond W. Profiles in patient safety: a "perfect storm" in the emergency department. Acad Emerg Med 2007;14:743-9.

15. Lakoff G, Johnson M. Philosophy in the flesh: the embodied mind and its challenge to Western thought. New York: Basic Books; 1999.
16. Hogarth RM. Educating intuition. Chicago: The University of Chicago Press; 2001.

17. Moulton CAE, Regehr G, Mylopoulos M, MacRae HM. Slowing down when you should: a new model of expert judgment. Acad Med 2007;82(10 Suppl):S109-16, doi: 10.1097/ACM.0b013e3181405a76.

18. Herbert W. On second thought: outsmarting your mind's hardwired habits. New York: Broadway Paperbacks; 2010.

19. Heath C, Heath D. Switch: how to change things when change is bard. New York: Broadway Paperbacks; 2010.

20. Wilson TD. Redirect; the surprising new science of psychological change. New York: Little, Brown and Company; 2011.

21. Stanovich KE. Rationality and the reflective mind. New York: Oxford University Press; 2011.

22. Guttmann A, Schull MJ, Vermeulen MJ, Stukel TA. Association between waiting times and short term mortality and hospital admission after departure from emergency department: population based cohort study from Ontario, Canada. BMF 2011;342:d2983, doi:10.1136/bmj.d2983.

23. Greene JD, Morelli SA, Lowenberg K, et al. Cognitive load selectively interferes with utilitarian moral judgment. Cognition 2008;107:1144-54.

24. Burgess DJ. Are providers more likely to contribute to healthcare disparities under high levels of cognitive load? How features of the healthcare setting may lead to biases in medical decision making. Med Decis Making 2010;30:246-57, doi:10.1177/0272989X09341751.

25. Hockey GRJ, Maule AJ, Clough PJ, Bdzola L. Effects of negative mood states on risk in everyday decision making. Cogn Emot 2000;14:823-55, doi:10.1080/02699930050156654.

26. Croskerry $\mathrm{P}$, Abbass A, Wu A. Emotional issues in patient safety. $\mathcal{f}$ Pat Saf 2010;6:1-7, doi:10.1097/PTS.0b013e 3181d321ca.

27. Croskerry P. Shiftwork, fatigue, and safety in emergency medicine. In: Croskerry P, Cosby KS, Schenkel S, Wears R, (editors). Patient safety in emergency medicine. Philadelphia: Lippincott Williams \& Wilkins; 2008. p. 259-68.

28. Straif K, Baan R, Grosse Y, et al. WHO International Agency for Research on Cancer Monograph Working Group. Carcinogenicity of shift-work, painting, and firefighting. Lancet Oncol 2007;8:1065-6, doi:10.1016/S14702045(07)70373-X.

29. Van Dongen HPA, Maislin G, Mullington JM, Dinges DF. The cumulative cost of additional wakefulness: doseresponse effects on neurobehavioral functions and sleep physiology from chronic sleep restriction and total sleep deprivation. Sleep 2003;26:117-26.

30. Danzigera S, Levavb J, Avnaim-Pessoa L. Extraneous factors in judicial decisions. Proc Natl Acad Sci U S A 2011;108:688992, doi:10.1073/pnas.1018033108.

31. Cox TK, Krueger G. Stress and sustained performance. Work Stress 1989;3:1-2, doi:10.1080/02678378908256874.

32. Krueger G. Sustained work, fatigue, sleep loss and performance: a review of the issues. Work Stress 1989;3: 121-41, doi:10.1080/02678378908256939.

33. Jones K, Harrison Y. Frontal lobe function, sleep loss and fragmented sleep. Sleep Med Rev 2001;5:463-75, doi:10.1053/ smrv.2001.0203. 